\title{
Degrees in random uniform minimal factorizations
}

\author{
Etienne Bellin * \\ CMAP - Ecole Polytechnique
}

\begin{abstract}
We are interested in random uniform minimal factorizations of the $n$-cycle which are factoriza-

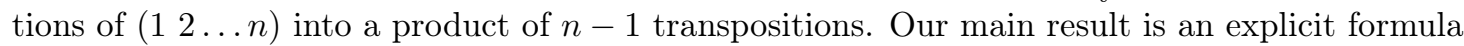
for the joint probability that 1 and 2 appear a given number of times in a uniform minimal factorization. For this purpose, we combine bijections with Cayley trees together with explicit computations of multivariate generating functions.
\end{abstract}

\section{Introduction}

Consider the cycle permutation $(12 \ldots n)$ for $n \geq 2$. A minimal factorization is a $(n-1)$-tuple of transpositions $\left(\tau_{1}, \ldots, \tau_{n-1}\right)$ such that $\tau_{n-1} \circ \cdots \circ \tau_{1}=(12 \ldots n)$. We denote by $\mathfrak{M}_{n}$ the set of all minimal factorizations of the cycle $(12 \ldots n)$. Dénes Dé59] first showed that $\mathfrak{M}_{n}$ has cardinality $n^{n-2}$ and several bijective proofs followed afterwards (see [Mos89, [GP93, GY02 and Bia05]). Minimal factorizations are linked to other combinatorial objects such as non-crossing partitions [Bia97, and parking functions [Bia02] and more general factorizations have deep connections with enumerative geometry (see e.g [ACEH18]).

Let $\left(\tau_{1}^{(n)}, \ldots, \tau_{n-1}^{(n)}\right)$ be a random minimal factorization chosen uniformly at random in $\mathfrak{M}_{n}$. The study of the behaviour of such a randomly picked minimal factorization is recent (see [FK18, [FK19] and [The20]) and has a rich probabilistic structure: for instance, it is shown in [The20] that such minimal factorizations have connections with Aldous-Pitman fragmentation of the Brownian continuum random tree. Here we are interested in the law of the number of times 1 and 2 appear in $\left(\tau_{1}^{(n)}, \ldots, \tau_{n-1}^{(n)}\right)$. In [FK19, Corollary 1.2 (iv)] it was obtained that:

$$
\mathbb{P}\left(\mathbb{T}_{1}^{(n)}=i, \mathbb{T}_{2}^{(n)}=j\right) \underset{n \rightarrow \infty}{\longrightarrow} e^{-2}\left[\frac{i+j-2}{(i+j-1) !}+\frac{i+j-1}{i ! j !}-\frac{i+j-1}{(i+j) !}\right] .
$$

where $\mathbb{T}_{k}^{(n)}=\#\left\{1 \leq \ell \leq n-1: \tau_{\ell}^{(n)}(k) \neq k\right\}$ is the number of time $k$ appears in a transposition. We refine this result by finding explicitly the joint distribution for fixed $n$ :

Theorem 1. For $i, j \geq 1$ and $n \geq i+j$ :

$$
\mathbb{P}\left(\mathbb{T}_{1}^{(n)}=i, \mathbb{T}_{2}^{(n)}=j\right)=\frac{n !(n-1)^{n-i-j-1}}{(n-i-j) !(n+1)^{n-1}}\left[\frac{(i+j-2)(n-1)}{(i+j-1) !(n-i-j+1)}+\frac{i+j-1}{i ! j !}-\frac{i+j-1}{(i+j) !}\right] .
$$

To show Theorem 1] we explicitly compute the exponential generating function of the (normalized) trivariate generating function $G_{n}$ defined by

$$
G_{n}(x, y, z)=n^{n-2} \mathbb{E}\left[x^{\mathbb{\Xi}_{1}^{(n)}} y^{\mathbb{\mathbb { V }}_{2}^{(n)}} z^{\mathbb{M}_{1}^{(n)}}\right]
$$

where $\mathbb{M}_{k}^{(n)}=\#\left\{1 \leq \ell \leq n-1: \tau_{1}^{(n)}(k) \ldots \tau_{\ell-1}^{(n)}(k) \neq \tau_{1}^{(n)}(k) \ldots \tau_{\ell}^{(n)}(k)\right\}$ is the number of transpositions that affect the trajectory of $k$, and then extract the coefficient $\left[x^{i} y^{j}\right] G_{n}(x, y, 1)$. To this end, there are 4 main steps. First, using a known bijection between minimal factorizations and Cayley trees, we reformulate the problem in terms of a generating function of a trivariate statistic on Cayley trees (Section 2). To compute this generating function, we actually start by computing another generating function $F_{n}$ obtained by changing one of the three statistics (Section 3). This also yields a result of independent interest by confirming a conjecture [Car19] involving distributional symmetries in uniform Cayley trees (Corollary 10). Finally, we show bijectively that $G_{n}(x, y, z)=G_{n}(y, x, z)$ (Section 4.1), and by combining this with the explicit formula of $F_{n}$ we get the exponential generating function of $G_{n}$ (Section 4.2) and Theorem 1 follows (Section 4.3).

\footnotetext{
*etienne.bellin@polytechnique.edu
} 


\section{Bijection between minimal factorizations and Cayley trees}

\subsection{The bijection}

Here we explain how to associate a labeled tree with a minimal factorization which will be an essential tool for us. We refer to [FK19] for details and proofs. Fix an integer $n \geq 2$.

Definition 2. For $\left(\tau_{1}, \ldots, \tau_{n-1}\right) \in \mathfrak{M}_{n}$, we define a labeled tree $\mathcal{F}\left(\tau_{1}, \ldots, \tau_{n-1}\right)$ with $n$ vertices labeled from 1 to $n$ where an edge labeled $l$ is drawn between the vertices labeled $a$ and $b$ if and only if $\tau_{l}=(a, b)$ (see figure 1 for an example).
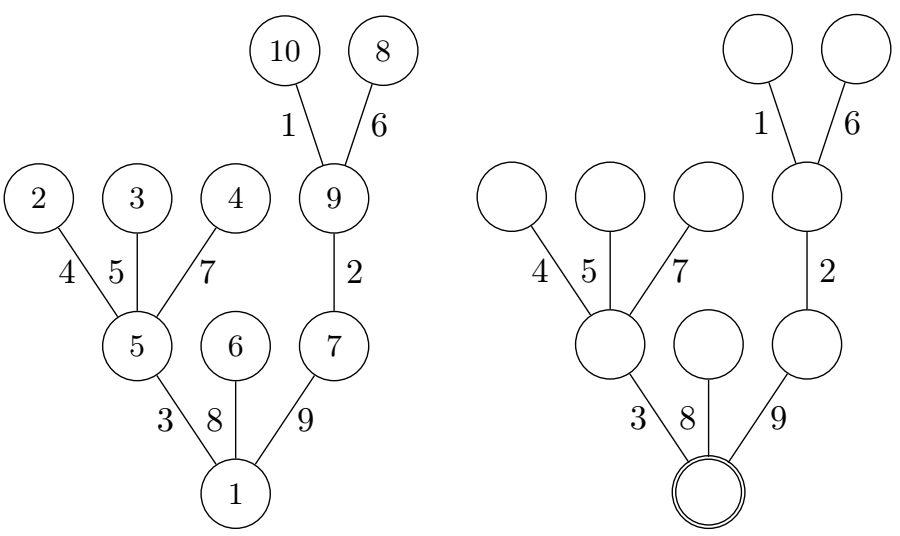

Figure 1: Representation of $\mathcal{F}$ on the left and $\mathcal{E}$ on the right when $n=10$ for the minimal factorization ((9 10), (7 9), (1 5), (2 5), (3 5), (8 9), (4 5), (1 6), (1 7)) of (1 . 10). The double circle represents the root of the tree $\mathcal{E}$.

Clearly $\mathcal{F}$ is injective since a minimal factorization can be easily read on its associated tree. Actually the tree $\mathcal{F}$ gives too much information, indeed it is still possible to retrieve the associated minimal factorization when we erase the vertex-labels and keep only the edge-labels. More precisely we have:

Definition 3. If $\left(\tau_{1}, \ldots, \tau_{n-1}\right) \in \mathfrak{M}_{n}$, then we construct a rooted, edge-labeled tree $\mathcal{E}\left(\tau_{1}, \ldots, \tau_{n-1}\right)$ by doing the following on the tree $\mathcal{F}\left(\tau_{1}, \ldots, \tau_{n-1}\right)$ :

- We root the tree at the vertex labeled 1.

- We erase all the vertex-labels (and keep only the edge-labels).

Proposition 4. The map $\mathcal{E}$ gives a bijection between the set $\mathfrak{M}_{n}$ and the set $\mathfrak{C}_{n}^{\prime}$ of rooted trees with $n-1$ edges labeled from 1 to $n-1$.

The set $\mathfrak{C}_{n}^{\prime}$ is clearly in bijection with the set $\mathfrak{C}_{n}$ of Cayley trees with $n$ vertices (i.e. trees with $n$ vertices labeled from 1 to $n$ ). Indeed if $t \in \mathfrak{C}_{n}$ we create $\alpha(t) \in \mathfrak{C}_{n}^{\prime}$ by rooting the tree $t$ at the vertex labeled 1 , then by pulling all the vertex-labels (except 1 which is erased from $\alpha(t)$ ) towards the root into the nearest edge. We then subtract 1 from all the labels (see figure 2 for an example). The map $\alpha$ is clearly a bijection.
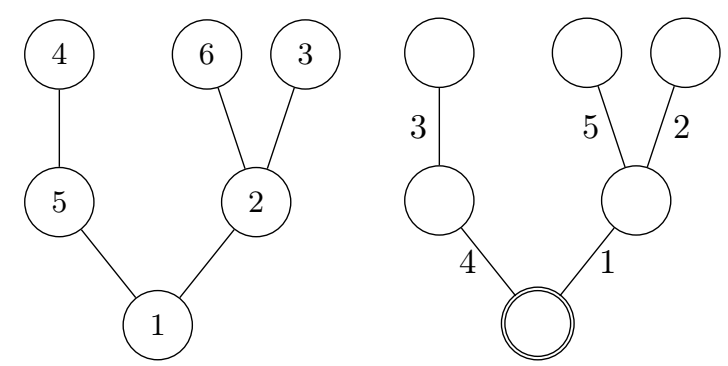

Figure 2: A tree $t \in \mathfrak{C}_{6}$ on the left transformed into $\alpha(t) \in \mathfrak{C}_{6}^{\prime}$ on the right by pulling the vertex-labels towards the root and subtracting 1 .

In particular $\mathfrak{M}_{n}$ has the same cardinality as the set of Cayley trees with $n$ vertices $\mathfrak{C}_{n}$ which is known to be $n^{n-2}$. This explains the renormalizing term in the definition of $G_{n}$. In the article [FK19] the authors 
give an explicit algorithm to find a minimal factorization back from its associated tree $\mathcal{E}$. We explain this algorithm right now because it will be essential for what follows.

Let $t \in \mathfrak{C}_{n}^{\prime}$ and $f \in \mathfrak{M}_{n}$ such that $t=\mathcal{E}(f)$. To recover $f$ from $t$, we will recover $\mathcal{F}(f)$ (from which it is immediate to find $f$ back). For this purpose, we will gradually assign the vertex-labels $1,2, \ldots, n$ to $t$ as they can be found in $\mathcal{F}(f)$. More precisely, let us describe an algorithm Next that takes the tree $t$ and an integer $k \in\{1, \ldots, n-1\}$ as arguments and assigns the vertex-label $k+1$ to $t$ if the vertex-label $k$ has already been assigned in $t$ (if $k$ is not assigned yet $\operatorname{Next}(\mathrm{t}, \mathrm{k})$ does nothing). The algorithm starts from the vertex $v_{0}$ of $t$ labeled $k$. Then it follows the longest possible path of edges $e_{1}, \ldots, e_{\ell}$ in $t$ such that:

- $e_{1}, \ldots, e_{\ell}$ is a path of edges meaning that for all $0<i<\ell, e_{i}$ and $e_{i+1}$ share a common vertex $v_{i}$.

- $e_{1}$ is the edge with smallest label adjacent to $v_{0}$.

- For all $0<i<\ell, e_{i+1}$ has the smallest label among the edges adjacent to $v_{i}$ having a label greater than $e_{i}$ 's label.

Starting from $v_{0}$ and following the path $e_{1}, \ldots, e_{\ell}$ leads to the vertex of $t$ which then gets the label $k+1$. We also initiate the algorithm with $\operatorname{Next}(t, 0)$ which gives label 1 to the root of $t$.

Now by applying successively Next to $t$ we can label all the vertices of $t$ in order to find back $\mathcal{F}(f)$. More precisely we have the following definition and Proposition:

Definition 5. For $1 \leq k \leq n$ and $t \in \mathfrak{C}_{n}^{\prime}$, we denote by Find $_{k}(t)$ the tree obtained by applying successively $\operatorname{Next}(t, 0)$ then $\operatorname{Next}(t, 1), \operatorname{Next}(t, 2), \ldots, \operatorname{Next}(t, k-1)$ to $t$.

For example, applying Find 10 to the tree on the right of figure 1 gives back the vertex-labels on the left of figure 1 .

Proposition 6. For $t=\mathcal{E}(f) \in \mathfrak{C}_{n}^{\prime}$ :

$$
\operatorname{Find}_{n}(t)=\mathcal{F}(f)
$$

\subsection{Reformulation in terms of Cayley trees}

In order to prove Theorem 1, we start with rewriting the generating function $G_{n}$ defined in the Introduction in terms of Cayley trees. To this purpose we introduce some notation. For $A$ a subset of $\{1, \ldots, n\}$ we denote by $\mathfrak{C}_{A}$ the set of trees with $|A|$ vertices labeled in a one-to-one manner with the elements of $A$. Notice that if $A=\{1, \ldots, n\}$ then $\mathfrak{C}_{A}=\mathfrak{C}_{n}$ is the set of Cayley trees with $n$ vertices. If $t \in \mathfrak{C}_{A}$ and $i \in A$, we denote by $\operatorname{deg}_{i}(t)$ the degree of the vertex $i$ (where "the vertex $i$ " has to be understood as "the vertex labeled $i$ ") in the tree $t$. Also, similarly to the algorithm Next (introduced in the previous Section) we consider a certain path in $t$ starting from $i$, namely:

Definition 7. For $t \in \mathfrak{C}_{A}$ and $i \in A$ we consider the longest path of vertices, starting from $i$, such that each vertex of the path has the smallest label among the ones that are both adjacent to the previous vertex on the path and have a greater label than the label of the previous vertex on the path. We denote by $L_{i}(t)$ the set of vertices composing this path but without including the first one which is the vertex $i$ (see figure 3 for an example).

Definition 8. For $t \in \mathfrak{C}_{n}$ we denote by $\operatorname{deg}_{2}^{\prime}(t)$ the degree, in $t$, of the last vertex of the path $L_{1}(t)$ (see figure 3 for an example).

Recall from Section 2.1 the bijection $\mathcal{E}$ between $\mathfrak{M}_{n}$ and $\mathfrak{C}_{n}^{\prime}$ as well as a bijection $\alpha$ from $\mathfrak{C}_{n}$ to $\mathfrak{C}_{n}^{\prime}$ (illustrated in figure 2). Notice that if $f \in \mathfrak{M}_{n}$ and $t=\alpha \circ \mathcal{E}(f)$ then

$$
\left(\mathbb{T}_{1}(f), \mathbb{T}_{2}(f), \mathbb{M}_{1}(f)\right)=\left(\operatorname{deg}_{1}(t), \operatorname{deg}_{2}^{\prime}(t),\left|L_{1}(t)\right|\right)
$$

where $\mathbb{T}_{i}(f)$ is the number of times $i$ appears in $f$ and $\mathbb{M}_{j}(f)$ is the number of transpositions in $f$ that affect the trajectory of $j$. The last identity allows us to reformulate the definition of $G_{n}$ for $n \geq 2$ :

$$
G_{n}(x, y, z)=\sum_{t \in \mathfrak{C}_{n}} x^{\operatorname{deg}_{1}(t)} y^{\operatorname{deg}_{2}^{\prime}(t)} z^{\left|L_{1}(t)\right|} .
$$

In the next Section, in order to compute $G_{n}$, we introduce another generating function $F_{n}$ whose definition is similar to (2) except that $\operatorname{deg}_{2}^{\prime}$ is replaced with $\operatorname{deg}_{2}$. 


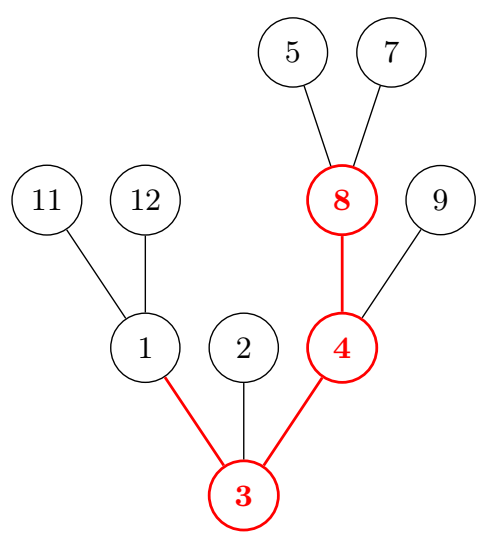

Figure 3: Example of a tree $t \in \mathfrak{C}_{A}$ with $A=\{1,2,3,4,5,7,8,9,11,12\}$. The path $L_{1}(t)=\{3,4,8\}$ starting from 1 is represented in thick red. Here $\operatorname{deg}_{2}^{\prime}(t)=\operatorname{deg}_{8}(t)=3$.

\section{First generating function on Cayley trees}

For $n \geq 2$ we define the generating function:

$$
F_{n}(x, y, z)=\sum_{t \in \mathfrak{C}_{n}} x^{\operatorname{deg}_{1}(t)} y^{\operatorname{deg}_{2}(t)} z^{\left|L_{1}(t)\right|} .
$$

By convention we also set for $n \geq 1$ :

$$
F_{n}(x, 1,1)=F_{n}(x)=\sum_{t \in \mathfrak{C}_{n}} x^{\operatorname{deg}_{1}(t)}=x(n-1+x)^{n-2} .
$$

The last equality is a well known result on Cayley trees. Actually, it turns out we have an explicit formula for $F_{n}(x, y, z)$.

Proposition 9. For $n \geq 3$,

$$
\begin{aligned}
F_{n}(x, y, z)=x y z & {\left[x(n-2+x)^{n-3}\left(1-\frac{y z}{z+y-1}\right)\right.} \\
+ & \left.(x+y+z+n-3)^{n-3}\left(n-2+y z+\frac{x y z}{z+y-1}\right)\right] .
\end{aligned}
$$

Formula (3) still holds in the cases $(y=1, n=2)$ and $(y=z=1, n=1)$. Proposition 9 implies that $F_{n}$ is symmetric in $y$ and $z$ thus we have the following Corollary which confirms a conjecture made by Caraceni Car19:

Corollary 10. Let $T_{n}$ be a random uniform Cayley tree with $n$ vertices, then $\left(\operatorname{deg}_{1}\left(T_{n}\right),\left|L_{1}\left(T_{n}\right)\right|, \operatorname{deg}_{2}\left(T_{n}\right)\right)$ and $\left(\operatorname{deg}_{1}\left(T_{n}\right), \operatorname{deg}_{2}\left(T_{n}\right),\left|L_{1}\left(T_{n}\right)\right|\right)$ have the same law.

It would be very interesting to obtain a direct bijective proof of Corollary 10. Formula (3) with $y=1$ was first conjectured in [FK19, Conjecture 1.4] and was proved by O. Angel \& J. Martin [AM19]. Below, we give Angel and Martin's proof of the case $y=1$ which will be useful to deduce the general case.

Proof of Proposition 9 for $y=1$. We show by induction on $n \geq 2$ that $F_{n}(x, 1, z)=x z f_{n}(x+z)$ where $f_{n}$ is a real-valued function. It is obviously true for $n=2$ with $f_{2}=1$. Suppose that is true for all $2 \leq k \leq n-1$ with $n \geq 3$.

We denote by $\mathcal{P}_{n}$ the set of couples $(A, B)$ with $A$ and $B$ two subsets of $\{1, \ldots, n\}$ such that $A \cup B=$ $\{1, \ldots, n\}, A \cap B=\varnothing, 1 \in A$ and $n \in B$. Let $t \in \mathfrak{C}_{n}$. Set the vertex 1 to be the root of $t$. Consider cutting the tree $t$ by removing the edge between the vertex $n$ and its parent to end up with two trees $t_{1} \in \mathfrak{C}_{A}$ and $t_{2} \in \mathfrak{C}_{B}$ for some $(A, B) \in \mathcal{P}_{n}$. Actually given two trees $t_{1} \in \mathfrak{C}_{A}$ and $t_{2} \in \mathfrak{C}_{B}$ with $(A, B) \in \mathcal{P}_{n}$ there are $|A|$ distinct ways to attach $t_{2}$ to $t_{1}$ by joining the vertex $n+1$ of $t_{2}$ to one of $t_{1}$ 's vertices to obtain a tree $t \in \mathfrak{C}_{n}$. With this in mind we can decompose the quantity $F_{n}(x, 1, z)$ depending on where $t_{2}$ is attached to $t_{1}$ :

$$
F_{n}(x, 1, z)=x z+\sum_{\substack{(A, B) \in \mathcal{P}_{n} \\|A|>1}} \sum_{\substack{t_{1} \in \mathfrak{C}_{A} \\ t_{2} \in \mathfrak{C}_{B}}}(|A|-2) x^{\operatorname{deg}_{1}\left(t_{1}\right)} z^{\left|L_{1}\left(t_{1}\right)\right|}+x^{\operatorname{deg}_{1}\left(t_{1}\right)+1} z^{\left|L_{1}\left(t_{1}\right)\right|}+x^{\operatorname{deg}_{1}\left(t_{1}\right)} z^{\left|L_{1}\left(t_{1}\right)\right|+1} .
$$


The first term corresponds to the case where $t_{1}$ has only 1 vertex (i.e. $|A|=1$ ). If $|A|>1$ then the vertex 1 and the last vertex of the path $L_{1}\left(t_{1}\right)$ are distinct in $t_{1}$ and we have to consider three cases: 1$)$ we attach $t_{2}$ to a vertex of $t_{1}$ which is neither 1 , nor the last vertex of $L_{1}\left(t_{1}\right)$. 2) We attach $t_{2}$ to the vertex 1 . 3) We attach $t_{2}$ to the last vertex of $L_{1}\left(t_{1}\right)$. We then have:

$$
F_{n}(x, 1, z)=x z+\sum_{a=2}^{n-1}\left(\begin{array}{l}
n-2 \\
a-1
\end{array}\right) F_{a}(x, 1, z)(a-2+x+z) .
$$

By induction we conclude that:

$$
F_{n}(x, 1, z)=x z+x z \sum_{a=2}^{n-1}\left(\begin{array}{l}
n-2 \\
a-1
\end{array}\right) f_{a}(x+z)(a-2+x+z) .
$$

So $F_{n}(x, 1, z) /(x z)$ depends only on $x+z$, thus induction is shown. We then just need to take $z=1$ and use the case $y=z=1$ to conclude.

To prove the general case of Proposition 9 we will use the particular cases $y=1$ and $z=1$. We will also use the following Abel's binomial Theorem [Rio79, p. 18].

Proposition 11. For every integer $n \geq 0$ the following identity holds:

$$
\sum_{k=0}^{n}\left(\begin{array}{l}
n \\
k
\end{array}\right) x(x-k z)^{k-1}(y+k z)^{n-k}=(x+y)^{n} .
$$

Three useful variants can be deduced from this identity.

Corollary 12. For every integer $n \geq 0$,

$$
\begin{array}{ll}
\text { Variant } 1 & \sum_{k=0}^{n}\left(\begin{array}{l}
n \\
k
\end{array}\right)(x+k)^{k-1}(y-k)^{n-k}=\frac{(x+y)^{n}}{x} ; \\
\text { Variant 2 } & \sum_{k=0}^{n}\left(\begin{array}{l}
n \\
k
\end{array}\right)(x+k)^{k}(n-k+y)^{n-k-1}=\frac{(x+y+n)^{n}}{y} ; \\
\text { Variant 3 } & \sum_{k=0}^{n}\left(\begin{array}{l}
n \\
k
\end{array}\right)(x+k)^{k-1}(n-k+y)^{n-k-1}=\frac{x+y}{x y}(x+y+n)^{n-1} .
\end{array}
$$

Proof. Taking $z=-1$ in Abel's binomial formula gives the first variant. Doing the change of index $k \rightarrow n-k$ and the change of variables $y \rightarrow x+n$ and $x \rightarrow y$ in variant 1 gives variant 2 . To get variant 3 we begin by differentiating variant 1 with respect to $y$, so we have:

$$
\sum_{k=0}^{n}\left(\begin{array}{l}
n \\
k
\end{array}\right)(n-k)(x+k)^{k-1}(n-k+y)^{n-k-1}=n \frac{(x+y+n)^{n-1}}{x} .
$$

Denote by $A$ the left side of variant 3 which we want to compute, then:

$$
n A-n \sum_{k=1}^{n}\left(\begin{array}{l}
n-1 \\
k-1
\end{array}\right)(x+k)^{k-1}(n-k+y)^{n-k-1}=n \frac{(x+y+n)^{n-1}}{x} .
$$

By doing the change of index $k \rightarrow k+1$ in the last sum and using variant 2 for the resulting sum we get:

$$
n A-n \frac{(x+y+n)^{n-1}}{y}=n \frac{(x+y+n)^{n-1}}{x} .
$$

The expression of $A$ can be deduced from the last display and thus variant 3 is shown.

Proof of Proposition 9 in the general case. Assume $n \geq 3$. Once again we will use a "tree-cutting" argument but instead of cutting at vertex $n$, we cut at vertex 2 . More precisely, we denote by $\mathcal{Q}_{n}$ the set of all couples $(A, B)$ with $A$ and $B$ two subsets of $\{1, \ldots, n\}$ such that $A \cup B=\{1, \ldots, n\}, A \cap B=\varnothing, 1 \in A$ and $2 \in B$. Once again, we decompose the quantity $F_{n}(x, y, z)$ depending on where $t_{2}$ is attached to $t_{1}$ :

$$
F_{n}(x, y, z)=\sum_{(A, B) \in \mathcal{Q}_{n}} \sum_{\substack{t_{1} \in \mathfrak{C}_{A} \\ t_{2} \in \mathfrak{C}_{B}}}\left[(|A|-1) x^{\operatorname{deg}_{1}\left(t_{1}\right)} y^{\operatorname{deg}_{2}\left(t_{2}\right)+1} z^{\left|L_{1}\left(t_{1}\right)\right|}+x^{\operatorname{deg}_{1}\left(t_{1}\right)+1} y^{\operatorname{deg}_{2}\left(t_{2}\right)+1} z^{\left|L_{2}\left(t_{2}\right)\right|+1}\right]
$$


The first term appearing after the sums corresponds to attaching $t_{2}$ to a vertex which is not 1 in $t_{1}$ and the second term corresponds to attaching $t_{2}$ to the vertex 1 . We then have:

$$
F_{n}(x, y, z)=\sum_{a=2}^{n-1}\left(\begin{array}{l}
n-2 \\
a-1
\end{array}\right)(a-1) y F_{a}(x, 1, z) F_{n-a}(y)+\sum_{a=1}^{n-1}\left(\begin{array}{c}
n-2 \\
a-1
\end{array}\right) x y z F_{a}(x) F_{n-a}(y, 1, z) .
$$

Now we can use the cases $y=1$ and $y=z=1$ to replace the occurrences of $F_{n}$ in the last display. Let's compute the first sum which we call $A_{n}(x, y, z)$, afterwards we will compute the second one, $B_{n}(x, y, z)$.

$$
\begin{aligned}
A_{n}(x, y, z) & =\sum_{a=2}^{n-1}\left(\begin{array}{c}
n-2 \\
a-1
\end{array}\right)(a-1) x y^{2} z(a-2+x+z)^{a-2}(n-a-1+y)^{n-a-2} \\
& =(n-2) \sum_{a=0}^{n-3}\left(\begin{array}{c}
n-3 \\
a
\end{array}\right) x y^{2} z(a+x+z)^{a}(n-a-3+y)^{n-a-4} \\
& =(n-2) x y z(x+y+z+n-3)^{n-3} .
\end{aligned}
$$

The second equality comes from the fact that $(a-1)\left(\begin{array}{l}n-2 \\ a-1\end{array}\right)=(n-2)\left(\begin{array}{l}n-3 \\ a-2\end{array}\right)$. The last equality comes from variant 2 of Abel's binomial formula. Now for $B_{n}(x, y, z)$ we need to be careful and isolate the case $a=n-1$ because formula (3) doesn't apply in the case $(y=1, n=1)$.

$$
\begin{aligned}
B_{n}(x, y, z)-x^{2} y z(x+n-2)^{n-3}= & \sum_{a=0}^{n-3}\left(\begin{array}{c}
n-2 \\
a
\end{array}\right) x^{2} y^{2} z^{2}(a+x)^{a-1}(n-a-3+y+z)^{n-a-3} \\
= & \sum_{a=0}^{n-4}\left(\begin{array}{c}
n-3 \\
a
\end{array}\right) x^{2} y^{2} z^{2}(a+1+x)^{a}(n-a-4+y+z)^{n-a-4} \\
& +\sum_{a=0}^{n-3}\left(\begin{array}{c}
n-3 \\
a
\end{array}\right) x^{2} y^{2} z^{2}(a+x)^{a-1}(n-a-3+y+z)^{n-a-3} . \\
= & \frac{x^{2} y^{2} z^{2}}{y+z-1}\left[(x+y+z+n-3)^{n-3}-(x+n-2)^{n-3}\right] \\
& +x y^{2} z^{2}(x+y+z+n-3)^{n-3} .
\end{aligned}
$$

The second equality comes from the fact that $\left(\begin{array}{c}n-2 \\ a\end{array}\right)=\left(\begin{array}{c}n-3 \\ a-1\end{array}\right)+\left(\begin{array}{c}n-3 \\ a\end{array}\right)$. The last one comes from variants 1 and 2 of Abel's binomial Theorem. The desired formula follows easily.

\section{A second generating function on Cayley trees and Proof of Theorem 1}

The goal now is to compute the exponential generating function of $G_{n}$ :

$$
\sum_{n \geq 1} \frac{G_{n+1}(x, y, z)}{n !} t^{n}
$$

Then by identifying coefficients in formula (6), we will be able to prove Theorem 11. The first step is to establish a symmetry property of $G_{n}$ with a bijective approach.

\subsection{A symmetry result}

Before computing the exponential generating function of $G_{n}$ we first state a useful symmetry result.

Proposition 13. For all $n \geq 2$ :

$$
G_{n}(x, y, z)=G_{n}(y, x, z)
$$

Proof. We will prove it by finding a bijection $\phi$ in $\mathfrak{M}_{n}$ which exchanges $\mathbb{T}_{1}$ and $\mathbb{T}_{2}$ and keeps $\mathbb{M}_{1}$ unchanged. For $1 \leq k \leq n$ we set $\gamma(k)=3-k \bmod n$ so $\gamma$ is a permutation and $\gamma^{-1}=\gamma$. For $\left(\tau_{1}, \ldots, \tau_{n-1}\right) \in \mathfrak{M}_{n}$ we define $\phi\left(\tau_{1}, \ldots, \tau_{n-1}\right)=\gamma \circ \tau_{1} \circ \cdots \circ \tau_{n-1} \circ \gamma$. Notice that for any transposition $\tau=(a b), \gamma \circ \tau \circ \gamma=(\gamma(a) \gamma(b))$ 
hence $\phi\left(\tau_{1}, \ldots, \tau_{n-1}\right)$ is a product of $n-1$ transpositions. To see that $\phi$ has the expected property, we interpret the action of $\phi$ on the tree $\mathcal{F}$. The tree $\mathcal{F} \circ \phi$ is obtained from $\mathcal{F}$ by relabelling the vertex-labels according to the permutation $\gamma$ and the edge-labels according to the permutation $i \mapsto n-i$. Such an edgerelabelling implies that $\mathrm{Find}_{n}$ reads through $\mathcal{E} \circ \phi$ in the exact opposite order than in $\mathcal{E}$. Thus we easily check that $\phi\left(\tau_{1}, \ldots, \tau_{n-1}\right)$ sends 2 on 3,3 on $4, \ldots, n$ on 1 and 1 on 2 so $\phi\left(\tau_{1}, \ldots, \tau_{n-1}\right)$ is a minimal factorization of $(1 \ldots n)$.

\subsection{Computation of the exponential generating function of the second generating function}

Before computing the exponential generating function of $G_{n}$ let's introduce the Lambert $W$ function (see e.g CJK97]). It is by definition the solution (in the sense of formal series) of $W(z) e^{W(z)}=z$. Using Lagrange inversion, one can show that $W(z)=\sum_{n \geq 1} \frac{(-n)^{n-1}}{n !} z^{n}$. Again, using Lagrange inversion, one can also compute:

$$
e^{-r W(-z)}=\left[\frac{-W(-z)}{z}\right]^{r}=\sum_{n \geq 0} \frac{r(n+r)^{n-1}}{n !} z^{n}
$$

with the convention that $0 \times 0^{-1}=1$. These properties of $W$ will be useful when proving Proposition 14 and Theorem 1 .

Proposition 14. The following identity on formal series holds:

$$
\begin{aligned}
e^{t}(y-x) \sum_{n \geq 1} \frac{G_{n+1}(x, y, z)}{n !} t^{n}= & \frac{x y z(x-1)}{x+z-1} e^{-y W(-t)}-\frac{x y z(y-1)}{y+z-1} e^{-x W(-t)} \\
& +\frac{x y z^{2}(y-x)}{(x+z-1)(y+z-1)} e^{-(x+y+z-1) W(-t)}
\end{aligned}
$$

Proof. Fix $n \geq 1$. As in the proof of Proposition 9 in the case $y=1$, we denote by $\mathcal{P}_{n+1}$ the set of couples $(A, B)$ with $A$ and $B$ two subsets of $\{1, \ldots, n+1\}$ such that $A \cup B=\{1, \ldots, n+1\}, A \cap B=\varnothing, 1 \in A$ and $n+1 \in B$. Then with a similar "tree-cutting" argument that led to (4) we have:

$$
\begin{aligned}
G_{n+1}(x, y, z)=x y z \sum_{t \in \mathfrak{C}_{n}^{\prime}} y^{\operatorname{deg}_{1}(t)}+\sum_{\substack{(A, B) \in \mathcal{P}_{n+1} \\
|A|>1}} \sum_{\substack{t_{1} \in \mathfrak{C}_{A} \\
t_{2} \in \mathfrak{C}_{B}}}\left[(|A|-2) x^{\operatorname{deg}_{1}\left(t_{1}\right)} y^{\operatorname{deg}_{2}^{\prime}\left(t_{1}\right)} z^{\left|L_{1}\left(t_{1}\right)\right|}\right. \\
+x^{\operatorname{deg}_{1}\left(t_{1}\right)+1} y^{\operatorname{deg}_{2}^{\prime}\left(t_{1}\right)} z^{\left|L_{1}\left(t_{1}\right)\right|} \\
\left.+x^{\operatorname{deg}_{1}\left(t_{1}\right)} y^{\operatorname{deg}_{n+1}\left(t_{2}\right)+1} z^{\left|L_{1}\left(t_{1}\right)\right|+1}\right] .
\end{aligned}
$$

The first term corresponds to the case where $t_{1}$ has only 1 vertex (i.e. $|A|=1$ ). If $|A|>1$ then the vertex 1 and the last vertex of the path $L_{1}\left(t_{1}\right)$ are distinct in $t_{1}$ and we have to consider three cases: 1$)$ we attach $t_{2}$ to a vertex of $t_{1}$ which is neither 1 , nor the last vertex of $L_{1}\left(t_{1}\right)$. 2) We attach $t_{2}$ to the vertex 1 . 3) We attach $t_{2}$ to the last vertex of $L_{1}\left(t_{1}\right)$. We then have:

$$
G_{n+1}(x, y, z)=x y z F_{n}(y)+\sum_{a=2}^{n}\left(\begin{array}{c}
n-1 \\
a-1
\end{array}\right)\left((a-2) G_{a}(x, y, z)+x G_{a}(x, y, z)+y z F_{a}(x, 1, z) F_{n+1-a}(y)\right)
$$

By Proposition 13, we get:

$$
G_{n+1}(x, y, z)=x y z F_{n}(x)+\sum_{a=2}^{n}\left(\begin{array}{l}
n-1 \\
a-1
\end{array}\right)\left[(a-2) G_{a}(x, y, z)+y G_{a}(x, y, z)+x z F_{a}(y, 1, z) F_{n+1-a}(x)\right]
$$

Now if we make the difference between the last two equations, we obtain:

$$
\begin{aligned}
0= & x y z\left(F_{n}(y)-F_{n}(x)\right)+(x-y) \sum_{a=2}^{n}\left(\begin{array}{c}
n-1 \\
a-1
\end{array}\right) G_{a}(x, y, z) \\
& +z \sum_{a=2}^{n}\left(\begin{array}{c}
n-1 \\
a-1
\end{array}\right)\left[y F_{a}(x, 1, z) F_{n+1-a}(y)-x F_{a}(y, 1, z) F_{n+1-a}(x)\right]
\end{aligned}
$$


If $n \geq 2$, by using the third variant of Abel's binomial formula and Proposition 9 we get:

$$
\begin{aligned}
\sum_{a=2}^{n}\left(\begin{array}{c}
n-1 \\
a-1
\end{array}\right) F_{a}(x, 1, z) F_{n+1-a}(y)= & x z \frac{x+y+z-1}{x+z-1}(x+y+z+n-2)^{n-2} \\
& -\frac{x y z(x+n-1)^{n-2}}{y+z-1}
\end{aligned}
$$

and

$$
\begin{aligned}
\sum_{a=2}^{n}\left(\begin{array}{c}
n-1 \\
a-1
\end{array}\right) F_{a}(y, 1, z) F_{n+1-a}(x)= & y z \frac{x+y+z-1}{y+z-1}(x+y+z+n-2)^{n-2} \\
& -\frac{x y z(y+n-1)^{n-2}}{x+z-1} .
\end{aligned}
$$

So, finally,

$$
0=(x-y) \sum_{a=2}^{n}\left(\begin{array}{l}
n-1 \\
a-1
\end{array}\right) G_{a}(x, y, z)+u_{n-1}(x, y, z),
$$

where

$$
\begin{aligned}
u_{n-1}(x, y, z)= & \frac{x y^{2} z(x-1)}{x+z-1}(y+n-1)^{n-2}-\frac{x^{2} y z(y-1)}{y+z-1}(x+n-1)^{n-2} \\
& +\frac{x y z^{2}(y-x)(x+y+z-1)}{(x+z-1)(y+z-1)}(x+y+z+n-2)^{n-2} .
\end{aligned}
$$

Thus, by Pascal's inversion formula,

$$
(y-x) G_{n+1}(x, y, z)=\sum_{k=0}^{n}\left(\begin{array}{l}
n \\
k
\end{array}\right)(-1)^{n-k} u_{k}(x, y, z) .
$$

Now define for $n \geq 0$ the polynomials $P_{n}$ by

$$
P_{n}(u)=\sum_{k=0}^{n} u\left(\begin{array}{l}
n \\
k
\end{array}\right)(-1)^{n-k}(k+u)^{k-1} .
$$

Their exponential generating function is given by

$$
\begin{aligned}
\sum_{n \geq 0} \frac{P_{n}(u)}{n !} t^{n} & =\sum_{k \geq 0} u t^{k}(k+u)^{k-1} \sum_{n \geq k}\left(\begin{array}{l}
n \\
k
\end{array}\right) \frac{(-1)^{n-k}}{n !} t^{n-k} \\
& =u e^{-t} \sum_{k \geq 0} \frac{(k+u)^{k-1}}{k !} t^{k} \\
& =e^{-t-u W(-t)}
\end{aligned}
$$

This, combined with (7), readily gives the desired result.

\subsection{Proof of Theorem 1}

To simplify notation, for $n \geq 2$, set

$$
p_{i, j}^{n}=\mathbb{P}\left(\operatorname{deg}_{1}\left(T_{n}\right)=i, \operatorname{deg}_{2}^{\prime}\left(T_{n}\right)=j\right)
$$

so that

$$
p_{i, j}^{n}=\frac{1}{n^{n-2}}\left[x^{i} y^{j}\right] G_{n}(x, y, 1) .
$$

Proof of Theorem 1. Fix $i, j \geq 1$. We take the formula of Proposition 14 with $z=1$ and divide it by $(y-x)$ to get:

$$
\begin{aligned}
e^{t} \sum_{n \geq 1} \frac{G_{n+1}(x, y, 1)}{n !} t^{n}= & \frac{x y}{y-x}\left[e^{-y W(-t)}-e^{-x W(-t)}\right]+\frac{1}{y-x}\left[x e^{-x W(-t)}-y e^{-y W(-t)}\right] \\
& +e^{-(x+y) W(-t)}
\end{aligned}
$$


We shall identify the coefficient in front of $x^{i} y^{j}$ (which is a polynomial in $t$ ) in this formula. On the left of this equality, the coefficient is:

$$
e^{t} \sum_{n \geq 1} p_{i, j}^{n+1} \frac{(n+1)^{n-1}}{n !} t^{n}
$$

Using the identity $y^{k}-x^{k}=(y-x)\left(y^{k-1}+y^{k-2} x+\cdots+x^{k-1}\right)$ we deduce the coefficient on the right:

$$
\frac{(-W(-t))^{i+j}}{i ! j !}+\frac{(-W(-t))^{i+j-1}}{(i+j-1) !}+\frac{(-W(-t))^{i+j}}{(i+j) !} \text {. }
$$

Therefore

$$
\sum_{n \geq 1} p_{i, j}^{n+1} \frac{(n+1)^{n-1}}{n !} t^{n}=e^{-t} \frac{(-W(-t))^{i+j}}{i ! j !}+e^{-t} \frac{(-W(-t))^{i+j-1}}{(i+j-1) !}+e^{-t} \frac{(-W(-t))^{i+j}}{(i+j) !}
$$

We now fix $n \geq i+j$ and identify the coefficient associated with $t^{n}$ in the above formula. Fix $1 \leq \ell \leq n$. By using formula (5) we obtain:

$$
\left[t^{n}\right] e^{-t}(-W(-t))^{\ell}=\ell \sum_{k=\ell}^{n} \frac{(-1)^{n-k}}{(n-k) !} \frac{k^{k-1-\ell}}{(k-\ell) !} .
$$

Recall the definition of $P_{n}$ in (8). Taking $y=z=1$ in equation (17) gives:

$$
G_{n+1}(x, 1,1)=F_{n+1}(x)=P_{n}(x+1)-P_{n}(1)
$$

In particular,

$$
P_{n}^{(\ell)}(x+1)=F_{n}^{(\ell)}(x)=x \frac{(n-1) !}{(n-1-\ell) !}(n+x)^{n-1-\ell}+\ell \frac{(n-1) !}{(n-\ell) !}(n+x)^{n-\ell}
$$

with the convention that $1 /(-1) !=0$. On the other hand, by definition of $P_{n}$,

$$
P_{n}^{(\ell)}(x)=x \sum_{k=\ell+1}^{n}(-1)^{n-k}\left(\begin{array}{l}
n \\
k
\end{array}\right) \frac{(k-1) !}{(k-1-\ell) !}(k+x)^{k-1-\ell}+\ell \sum_{k=\ell}^{n}(-1)^{n-k}\left(\begin{array}{l}
n \\
k
\end{array}\right) \frac{(k-1) !}{(k-\ell) !}(k+x)^{k-\ell} .
$$

We finally obtain:

$$
\ell \sum_{k=\ell}^{n} \frac{(-1)^{n-k}}{(n-k) !} \frac{k^{k-1-\ell}}{(k-\ell) !}=\frac{1}{n !} P_{n}^{(\ell)}(0)=(n-1)^{n-\ell-1} \frac{\ell-1}{(n-\ell) !} .
$$

Formula (1) then follows.

\section{References}

[ACEH18] A Alexandrov, G Chapuy, B Eynard, and J Harnad. Weighted Hurwitz numbers and topological recursion: An overview. Journal of Mathematical Physics, 59(8):081102, Aug 2018.

[AM19] O Angel and J Martin. 14th probability and combinatorics workshop. Private communication, Barbados 2019.

[Bia97] P Biane. Some properties of crossings and partitions. Discrete Math., 175:41-53, 1997.

[Bia02] P Biane. Parking functions of types A and B. Electron. J. Combin., 9, 2002.

[Bia05] P Biane. Nombre de factorisations d'un grand cycle. Sem. Lothar. Combin., 51, 2004/2005.

[Car19] A Caraceni. 14th probability and combinatorics workshop. Private communication, Barbados 2019.

[CJK97] R Corless, D Jeffrey, and D Knuth. A sequence of series for the lambert $w$ function. ISSAC '97: Proceedings of the $199^{r}$ international symposium on Symbolic and algebraic computation, pages 197-204, 1997. 
[Dé59] J Dénes. The representation of a permutation as the product of a minimal number of transpositions, and its connection with the theory of graphs. Magyar Tud. Akad. Mat. Kutato Int. Kozl., 4:63-71, 1959.

[FK18] V Féray and I Kortchemski. The geometry of random minimal factorizations of a long cycle via biconditioned bitype random trees. Ann. H. Lebesgue, 1:149-226, 2018.

[FK19] V Féray and I Kortchemski. Trajectories in random minimal transposition factorizations. Lat. Am. J. Probab. Math. Stat., 16:759-785, 2019.

[GP93] I P Goulden and S Pepper. Labelled trees and factorizations of a cycle into transpositions. Discrete Math., 113:263-268, 1993.

[GY02] I Goulden and A Yong. Tree-like properties of cycle facorizations. J. Combinatorial Theory ser. A, 98:106-117, 2002.

[Mos89] P Moszkowski. A solution to a problem of denes: a bijection between trees and factorizations of cyclic permutations. European J. Combin., 10:13-16, 1989.

[Rio79] J Riordan. Combinatorial Identities. New York: Wiley, 1979.

[The20] $\mathrm{P}$ Thevenin. A geometric representation of fragmentation processes on stable trees. arXiv:1910.04508, 2020. 